American Chemical Society, J. Phys. Chem. C, jp072067f, Supporting Information Page S1

\title{
Large Pore Volume Carbons with Uniform Mesopores and Macropores: Synthesis, Characterization and Relations between Parameters of Silica Templates and their Inverse Carbon Replicas
}

\author{
Kamil P. Gierszal and Mietek Jaroniec \\ Department of Chemistry, Kent State University, Kent, Ohio, 44240 USA
}

\section{Derivation of the equations used in the paper:}

Notation:

$\mathrm{d}_{\mathrm{s}}\left[\mathrm{g} / \mathrm{cm}^{3}\right] \quad$ - density of amorphous silica $\left(2.2 \mathrm{~cm}^{3} / \mathrm{g}\right)$

$\mathrm{d}_{\mathrm{c}}\left[\mathrm{g} / \mathrm{cm}^{3}\right] \quad$ - density of amorphous carbon $\left(1.5 \mathrm{~cm}^{3} / \mathrm{g}\right.$ for phenolic resin-based carbon)

$\mathrm{S}_{\mathrm{S}}\left[\mathrm{m}^{2} / \mathrm{g}\right] \quad$ - specific surface area of a porous silica template

$\mathrm{S}_{\mathrm{c}}\left[\mathrm{m}^{2} / \mathrm{g}\right] \quad$ - specific surface area of an inverse carbon replica

$\mathrm{t}_{\mathrm{c}}[\mathrm{m}] \quad-$ thickness of pore walls in the carbon replica

$t_{c(\max )}^{-} \quad$ average maximum carbon film/wall thickness for the inverse carbon replica obtained by complete filling of pores in the silica template

$\mathrm{V}_{\mathrm{s}}\left[\mathrm{cm}^{3} / \mathrm{g}\right] \quad$ - specific pore volume of a porous silica template

$\mathrm{V}_{\mathrm{sc}}\left[\mathrm{cm}^{3} / \mathrm{g}\right] \quad$ - specific pore volume of a carbon-silica composite

$\mathrm{V}_{\mathrm{c}}\left[\mathrm{cm}^{3} / \mathrm{g}\right] \quad$ - specific pore volume of an inverse carbon replica

$\mathrm{V}_{\mathrm{c}(\min )}\left[\mathrm{cm}^{3} / \mathrm{g}\right]$ - minimum pore volume of an inverse carbon replica obtained by complete filling of pores in the silica template

$\mathrm{w}_{\text {coll }}[\mathrm{nm}] \quad$ - diameter of silica colloids

$\mathrm{x}$ [-] - ratio of the solid volume to the total volume of solid and pores (in the case of colloidal silica templates, x can be referred as "packing degree”)

Equation 1. Ratio of the solid (skeleton) volume to the total volume of solid and pores, called the packing degree, $x$.

Specific volume of silica, i.e., the volume of skeleton per gram of silica, is equal to the reciprocal true silica density:

$1 / d_{s}$

Taking into account the definition of the specific pore volume of a porous solid (i.e., the volume of pores per gram of the solid) one can express the total volume of a porous silica (skeleton with pores) as follows: 
American Chemical Society, J. Phys. Chem. C, jp072067f, Supporting Information Page S2

$V_{s}+1 / d_{s}$

Thus

$x=\frac{1 / d_{s}}{V_{s}+1 / d_{s}}$

Rearrangement of the above equation gives:

$x=\frac{1}{V_{s} \cdot d_{s}+1}$

Note that 1-x after multiplication by 100 denotes the well-known percentage porosity of a porous solid.

Equation 2. Specific surface area of a colloidal silica template, $\mathrm{S}_{\mathrm{s}}$.

Volume of one colloidal sphere:

$V_{\text {coll }}=\frac{1}{6} \pi\left(w_{\text {coll }}\right)^{3}$

Mass of one colloidal sphere:

$m_{\text {coll }}=V_{\text {coll }} \cdot d_{s}=\frac{1}{6} \pi\left(w_{\text {coll }}\right)^{3} \cdot d_{s}$

Surface area of one colloidal sphere:

$S_{\text {coll }}=\pi\left(w_{\text {voll }}\right)^{2}$

Specific surface area of colloidal silica, i.e., surface area per gram of silica, is given by:

$S_{s}=S_{\text {coll }} / m_{\text {coll }}=\frac{6}{w_{\text {coll }} \cdot d_{s}}$

Equation 3. Maximum average carbon film (wall) thickness, $t_{c(\max )}$, of an inverse carbon replica obtained by using a porous silica template.

Equation 3, $t_{c(\max )}=\frac{V_{s}}{S_{s}}$, can be derived by equalization of equations 4 and 5. 
Equation 4: $V_{c(\text { min) }}=\frac{1}{V_{s} \cdot d_{s} \cdot d_{c}}$ (see derivation below)

Equation 5: $V_{c}=\frac{V_{s}+\frac{1}{d_{s}}-S_{s} \cdot t_{c}}{S_{s} \cdot t_{c} \cdot d_{c}}$

Derivation of equation 5 can be found in the supporting information of our recent communication published in J. Am. Chem. Soc. 2006, 128, 10026.

The thickness of spherical pore walls in the carbon replica can be estimated by using the rearranged form of equation 5 :

$$
t_{c}=\frac{V_{s}+\frac{1}{d_{s}}}{S_{s} d_{c}\left(V_{c}+\frac{1}{d_{c}}\right)}
$$

Equation 4. Minimum total pore volume of an inverse carbon replica obtained using a porous silica template, $\mathrm{V}_{\mathrm{c}(\mathrm{min})}$. This quantity denotes the specific pore volume of the inverse carbon replica obtained via complete filling of all pores in the silica template with carbon followed by the template dissolution.

The volume of the aforementioned pores expressed per gram of the silica template is equal to the volume of the silica skeleton, $1 / \mathrm{d}_{\mathrm{s}}$. To express this volume of pores per gram of the carbon replica one needs to determine the carbon mass in the completely filled pores of $1 \mathrm{~g}$ silica template. This carbon mass can be obtained by multiplying the specific pore volume of the silica template, $\mathrm{V}_{\mathrm{s}}$, by the true carbon density, $\mathrm{d}_{\mathrm{c}}$.

Thus, the minimum specific pore volume of an inverse carbon replica, $\mathrm{V}_{\mathrm{c}(\min )}$, can be expressed as follows:

$V_{c(\text { min })}=\frac{\frac{1}{d_{s}}}{V_{s} d_{c}}=\frac{1}{V_{s} \cdot d_{c} \cdot d_{s}}$

Equation 7. Total specific pore volume of the carbon-silica composite, $\mathrm{V}_{\mathrm{sc}}$.

In the case of an incomplete filling of the silica template pores with carbon, the unoccupied volume of pores in $1 \mathrm{~g}$ of the silica template is equal to: 
American Chemical Society, J. Phys. Chem. C, jp072067f, Supporting Information Page S4

$V_{s}-S_{s} t_{c}$

The total mass of the silica-carbon composite obtained by using $1 \mathrm{~g}$ of the silica template is a simple sum of $1 \mathrm{~g}$ of the silica and the carbon mass present in the pores of $1 \mathrm{~g}$ silica, $\mathrm{S}_{\mathrm{s}} \mathrm{t}_{\mathrm{c}} \mathrm{d}_{\mathrm{c}}$, i.e.,

$S_{s} t_{c} d_{c}+1$

Thus, the specific pore volume of the silica-carbon composite is:

$V_{s c}=\frac{V_{s}-S_{s} \cdot t_{c}}{S_{s} \cdot t_{c} \cdot d_{c}+1}$

Thus, the carbon film thickness (which is equivalent to the thickness of the pore walls in the carbon replica) can be estimated on the basis of the specific pore volume of the silica-carbon composite by rearranging equation for $\mathrm{V}_{\mathrm{sc}}$ :

$$
t_{c}=\frac{V_{s}-V_{s c}}{S_{s}\left(V_{s c} \cdot d_{c}+1\right)}
$$

Equation 8. Specific surface area $\left(\mathrm{S}_{\mathrm{c}}\right)$ of a film-type inverse carbon replica. In this case the surface area of an inverse carbon replica is twice greater than the surface area obtained by dividing the volume of the carbon skeleton, $1 / \mathrm{d}_{\mathrm{c}}$, by the carbon film thickness, $\mathrm{t}_{\mathrm{c}}$ :

$$
S_{c}=\frac{2}{t_{c} \cdot d_{c}}
$$

Equation 9. Specific surface area of an inverse carbon replica obtained by complete filling of pores in the silica template. This quantity can be obtained by dividing the volume of the carbon skeleton, $1 / d_{c}$, by the carbon film thickness, $t_{c}$ :

$$
S_{c}=\frac{1}{t_{c} \cdot d_{c}}
$$

Substitution of $\mathrm{t}_{\mathrm{c}}$ by equation 3 gives:

$$
S_{c}=\frac{S_{s}}{V_{s} \cdot d_{c}}
$$


American Chemical Society, J. Phys. Chem. C, jp072067f, Supporting Information Page S5

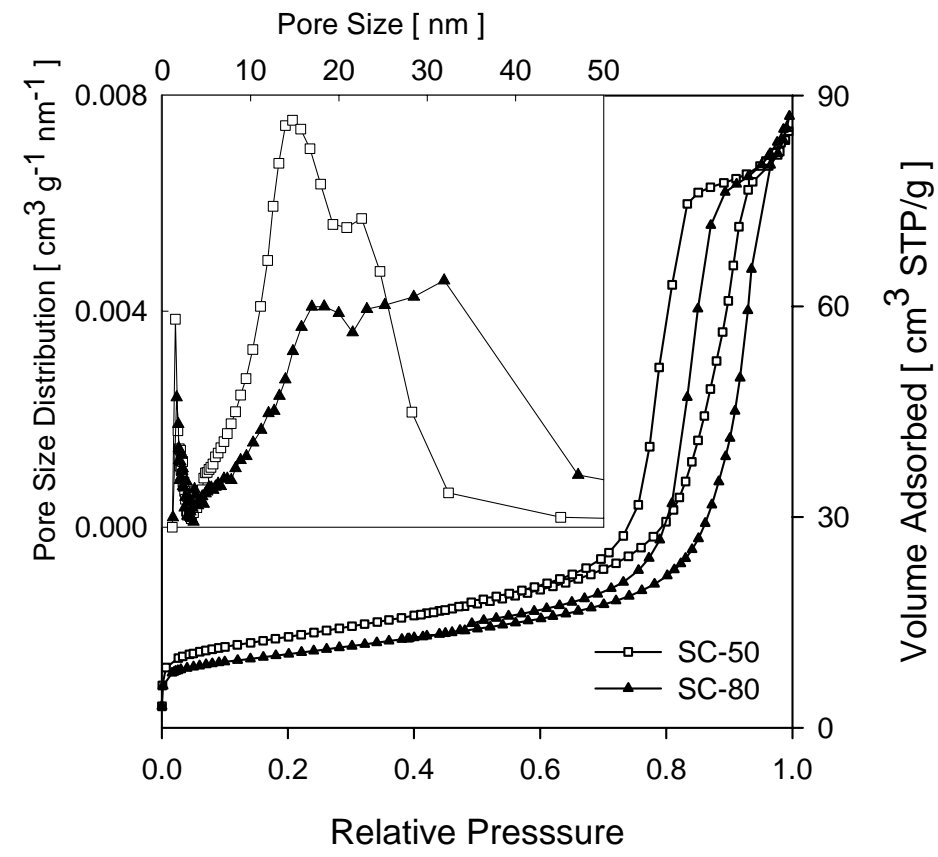

Figure 1S. Nitrogen adsorption isotherms at $-196{ }^{\circ} \mathrm{C}$ and the corresponding pore size distributions for the silica-carbon composites obtained by carbonization (at $900{ }^{\circ} \mathrm{C}$ ) of resorcinolcrotonaldehyde film formed on the surface of 50 and $80 \mathrm{~nm}$ silica colloids.

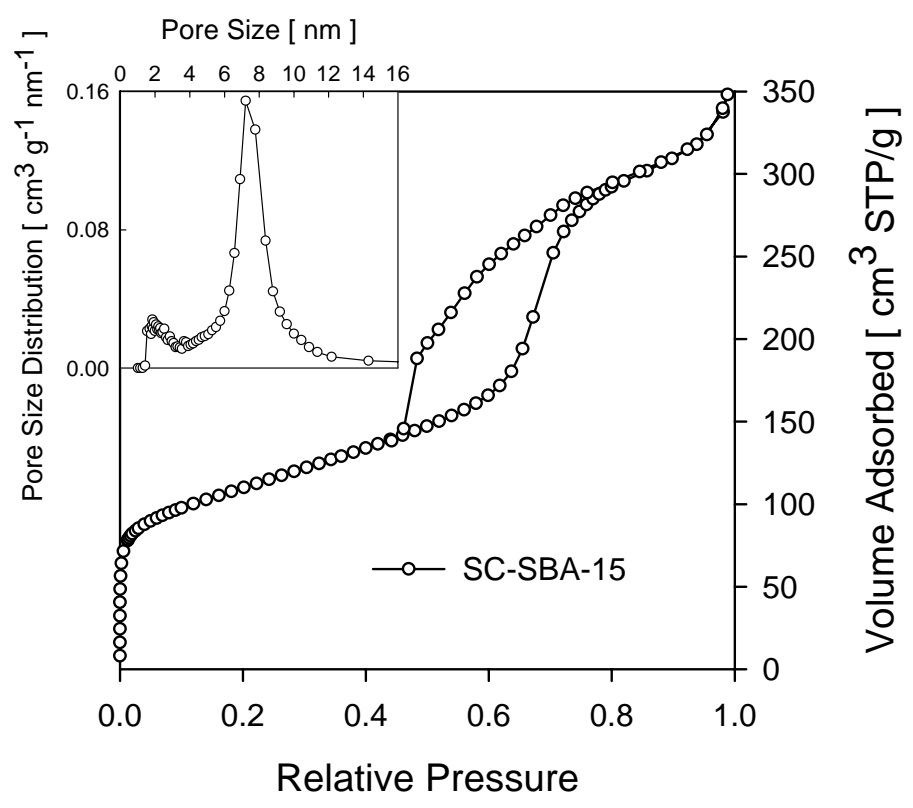

Figure 2S. Nitrogen adsorption isotherm at $-196{ }^{\circ} \mathrm{C}$ and the corresponding pore size distribution for the carbon-silica composite obtained by carbonization (at $900{ }^{\circ} \mathrm{C}$ ) of resorcinolcrotonaldehyde film formed on the pore walls of the SBA-15 silica template. 


\section{Adsorption and structural properties of the additional silica and carbon samples}

The silica templates listed in Table $1 \mathrm{~S}$ are the same as those in the paper except S-22, which was obtained by using $22 \mathrm{~nm}$ silica colloids. The carbon samples listed in Table $2 \mathrm{~S}$ were synthesized in the same way as those described in the paper except the amount of the resorcinol used. The experimental differences and additional information are provided below:

The S-22 silica template was synthesized by centrifugation of commercial monodisperse spherical silica colloids having diameter of $22 \mathrm{~nm}$ (Ludox AS-40 provided by the W. R. Grace \& Co. - Davison; an aqueous colloidal dispersion containing $40 \mathrm{wt} \%$ of silica stabilized with ammonium hydroxide at $\mathrm{pH}$ 9-10). Before centrifugation, aqueous colloidal dispersion Ludox AS-40 was mixed with $100 \%$-ethanol to achieve the $1: 10$ weight ratio.

The carbon replica of the S-22 silica template was obtained by using $150 \mathrm{mg}$ resorcinol per 1 g silica, while another replica of the S-50 silica template was prepared by using $63 \mathrm{mg}$ resorcinol per $1 \mathrm{~g}$ silica. However, two carbon replicas of the S-SBA-15 template (denoted as C-SBA-15A and C-SBA-15B) were synthesized by using 450 and $635 \mathrm{mg}$ resorcinol per $1 \mathrm{~g}$ silica, respectively.

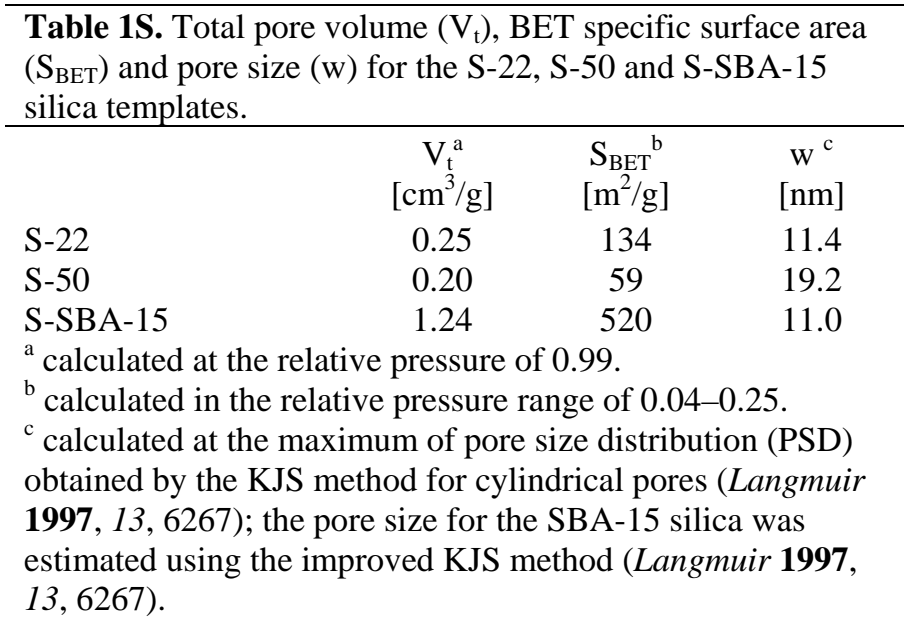

\footnotetext{
Table 2S. Total pore volume $\left(\mathrm{V}_{\mathrm{t}}\right)$, BET specific surface area $\left(\mathrm{S}_{\mathrm{BET}}\right)$, theoretical specific surface area $\left(\mathrm{S}_{\mathrm{c}}\right.$; eq 8), pore widths $(\mathrm{w})$ and theoretical carbon wall thickness ( $\mathrm{t}_{\mathrm{c}}$; eq 5 ) for the carbon replicas obtained by using S-22, S-50 and S-SBA-15 silicas as hard templates.

\begin{tabular}{|c|c|c|c|c|c|c|}
\hline Sample & $\begin{array}{c}\mathrm{V}_{\mathrm{t}}^{\mathrm{a}} \\
{\left[\mathrm{cm}^{3} / \mathrm{g}\right]}\end{array}$ & $\begin{array}{c}\mathrm{S}_{\mathrm{BET}}{ }^{\mathrm{b}} \\
\left(80 \% \mathrm{~S}_{\mathrm{BET}}\right) \\
{\left[\mathrm{m}^{2} / \mathrm{g}\right]}\end{array}$ & $\begin{array}{c}\mathrm{S}_{\mathrm{c}}{ }^{\mathrm{c}} \\
{\left[\mathrm{m}^{2} /\right.} \\
\mathrm{g}]\end{array}$ & $\frac{S_{c}-0.8 \cdot S_{B E T}}{0.8 \cdot S_{B E T}} \cdot 100 \%$ & $\begin{array}{l}\mathrm{w}^{\mathrm{d}} \\
{[\mathrm{nm}]}\end{array}$ & $\begin{array}{c}\mathrm{t}_{\mathrm{c}} \\
{[\mathrm{nm}]}\end{array}$ \\
\hline $\mathrm{C}-22$ & 4.35 & $1820(1456)$ & 1524 & 4.7 & $5.2 ; 23.8$ & 0.875 \\
\hline C- $50^{*}$ & 6.5 & 1330 (1064) & 1029 & -3.3 & 11.2 & 0 \\
\hline C-SBA-15A & 2.7 & 1960 (1568) & 1653 & 5.4 & & 0.81 \\
\hline C-SBA-15B & 2.45 & $1700(1360)$ & 1530 & 12.5 & $4.8 ; 7.8$ & 0.87 \\
\hline \multicolumn{7}{|c|}{$\begin{array}{l}\text { a calculated at the relative pressure of } 0.99 \text {; } \\
\text { b calculated in the relative pressure range of } 0.04-0.25 \text { (the values in brackets refer to } \mathrm{S}_{\mathrm{BET}} \text { reduced by } \\
20 \% \text { ); } \\
\text { c calculated by eq } 8 \text { using the carbon density of } 1.5 \mathrm{~cm}^{3} / \mathrm{g} \text {; } \\
\text { d pore widths estimated at the maxima of both peaks of bimodal pore size distribution (PSD); } \\
\text { * denotes different sample than that reported in Table } 2 \text { of the paper. Also, C-SBA-15A and B carbon } \\
\text { replicas denote different samples than that in Table } 2 \text {. }\end{array}$} \\
\hline
\end{tabular}
}


American Chemical Society, J. Phys. Chem. C, jp072067f, Supporting Information Page S7

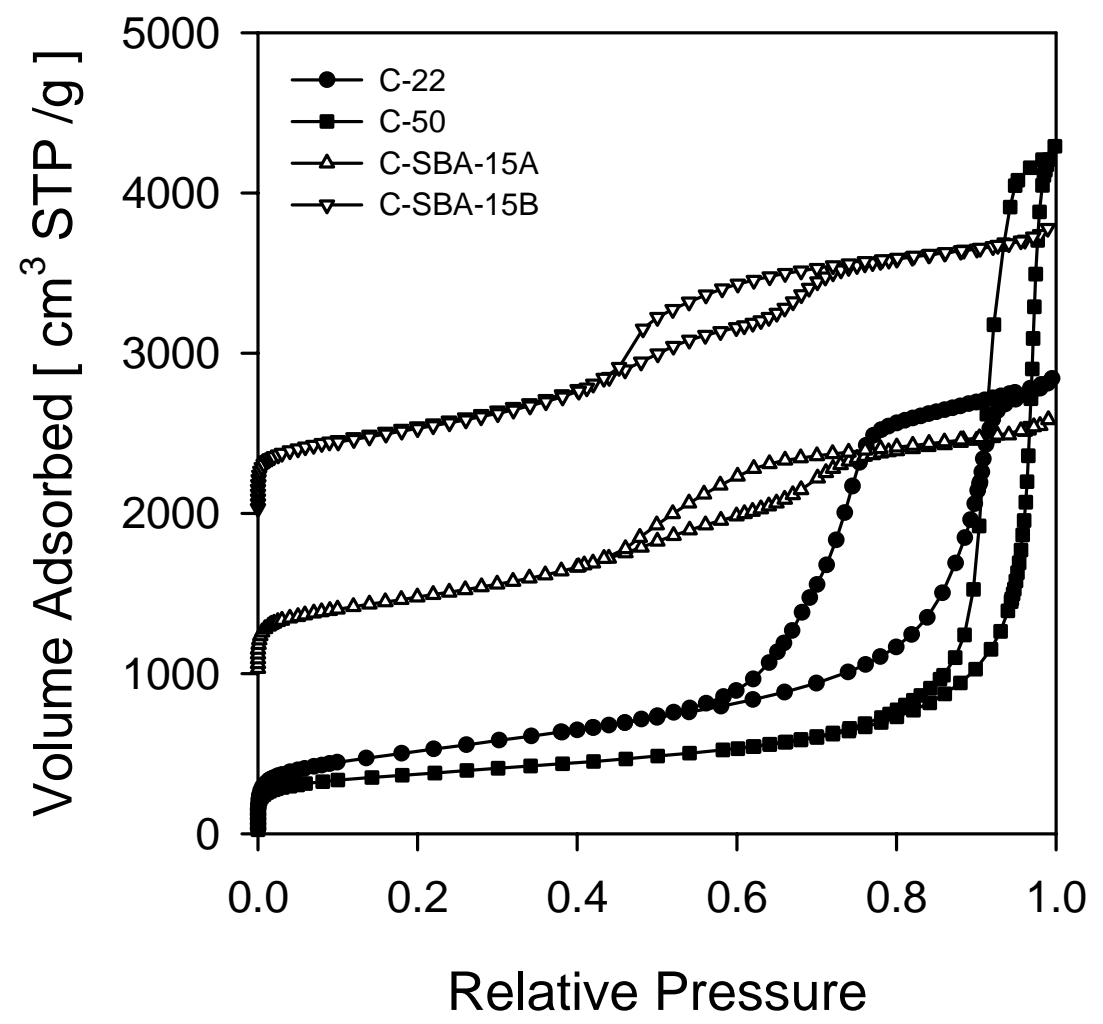

Figure 3S. Nitrogen adsorption isotherms at $-196{ }^{\circ} \mathrm{C}$ for the carbon samples obtained by carbonization (at $900{ }^{\circ} \mathrm{C}$ ) of the S-22, S-50 and S-SBA-15 silica templates coated with resorcinol-crotonaldehyde film followed by the silica dissolution. The isotherms for the C-SBA15A and C-SBA-15B are shifted vertically by 1000 and $2000 \mathrm{~cm}^{3}$ STP/g, respectively. 